\title{
Contribution of mathematical modelling to the interpretation of bedside tests of cerebrovascular autoregulation
}

Marek Czosnyka, Stefan Piechnik, Hugh K Richards, Peter Kirkpatrick, Piotr Smielewski, John D Pickard

\begin{abstract}
Objectives-Cerebral haemodynamic responses to short and longlasting episodes of decreased cerebral perfusion pressure contain information about the state of autoregulation of cerebral blood flow. Mathematical simulation may help to elucidate which of the indices, that can be derived using transcranial Doppler ultrasonography and trends of intracranial pressure and blood pressure, are useful in clinical tests of autoregulatory reserve.

Methods-Time dependent interactions between pressure, flow, and volume of cerebral blood and CSF were modelled using a set of non-linear differential equations. The model simulates changes in arterial blood inflow and storage, arteriolar and capillary blood flow controlled by cerebral autoregulation, venous blood storage and venous outflow modulated by changes in ICP, and CSF storage and reabsorption. The model was used to simulate patterns of blood flow during either short or longlasting decreases in cerebral perfusion pressure. These simulations can be considered as clinically equivalent to a short compression of the common carotid artery, systemic hypotension, and intracranial hypertension. Simulations were performed in autoregulating and nonautoregulating systems and compared with recordings obtained in patients.

Results-After brief compression of the common carotid artery, a subsequent transient hyperaemia can be interpreted as evidence of intact autoregulation. During longlasting sustained hypoperfusion, a gradual increase in the systolic value of the blood flow velocity waveform along with a decrease in the diastolic value is specific for an autoregulating cerebrovascular system.
\end{abstract}

Conclusion-Modelling studies help to interpret both clinical and experimental cerebral haemodynamic phenomena and their dependence on the state of autoregulation.

(F Neurol Neurosurg Psychiatry 1997;63:721-731)

Keywords: autoregulation, trascranial Doppler, intracranial pressure, mathematical modelling
The final outcome after injury to the brain depends not only on the initial trauma but also on secondary insults including, for example, raised intracranial pressure, arterial hypotension, and hypoxia. ${ }^{1}$ Autoregulation of cerebral blood flow (CBF) is an important factor in the brain's capacity to respond to such insults. ${ }^{23}$ Disordered autoregulation is a prelude to refractory intracranial hypertension and correlates with a poor outcome. ${ }^{4-6}$ The capacity for autoregulation differs between patients and varies in the same patient from day to day. ${ }^{6}$ Therefore, reliable and repeatable bedside techniques are required to monitor the variations in autoregulatory reserve if this is to be useful guide to therapy. ${ }^{4-9}$ However, the tests that can be used clinically explore only a small part of the range of the autoregulatory response and also depend on techniques such as waveform analysis of transcranial Doppler ultrasonography (TCD) and intracranial pressure $^{610}$ that have a complex relation with $\mathrm{CBF}$. The data can be difficult to interpret, especially in deciding if autoregulation is intact or impaired.

Experimental studies are advancing the ability to understand findings in patients but cannot replicate all aspects of the clinical problem. ${ }^{11-14}$

We have therefore developed a mathematical model of cerebrovascular flow which embeds previous models of CSF circulation ${ }^{15-17}$ to simulate interactions between $\mathrm{CBF}$ and intracranial pressure. ${ }^{18} 19$ The model incorporates realistic values for known components including cerebrovascular resistance, intracranial and arterial compliance, and CSF outflow resistance. $^{2021}$ This paper presents our experience in using the model to enhance the interpretation of bedside tests of cerebrovascular autoregulation.

\section{Materials and methods}

MODEL

The first models of the mechanoelastic properties of the brain were related to the CSF circulation and included the non-linear pressurevolume relation of the intracranial compartment. ${ }^{15182022}$ These studies laid the foundation for techniques used in the diagnosis of hydrocephalus today. ${ }^{172324}$ Advances in computing technology have prompted attempts to integrate the intracranial CSF circulation with $\mathrm{CBF}^{25-27}$

The pressure of CSF results from the dynamic equilibrium between the volumetric 


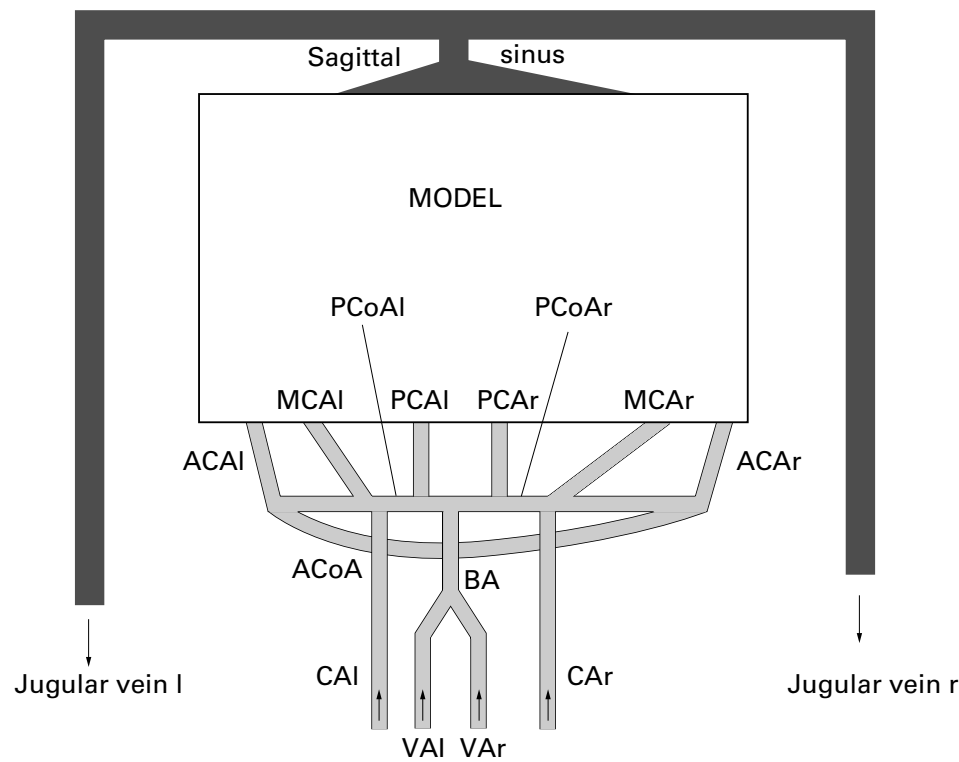

Figure 1 The anatomically equivalent location of the model is distal to the circle of Willis and proximal to the jugular veins. $P C o A=$ posterial communicating artery; $P C A=$ posterior cerebral artery; $V A=$ vertebral artery; $C A=$ carotid artery; $B=$ basilar artery; $A C A=$ anterior cerebral artery; $A C o A=$ anterior communicating artery.

changes in brain tissue, CSF volume, and cerebral blood volume. ${ }^{15} 1920$ Any deviation may generate a pressure response affecting the cerebral circulation with several stabilising feedback loops involved. These include cerebrovascular autoregulation/reactivity, neurogenic innervation, intraparenchymal water fluxes, and secretion/reabsorption of CSF. The model we propose contains two major flow pathways (figs 1 and 2). The CBF pathway starts with the arterial blood inflow to the brain through the resistance of large intracranial arteries $\left(\mathrm{R}_{\mathrm{a}}\right)$. At present, the model is unable to simulate phenomena resulting from the complex topography of the basal cerebral arteries included in the circle of Willis (fig 1). Arterial blood is contained in a high pressure arterial compliance $\mathrm{C}_{\mathrm{a}}$. Forward flow through the cerebrovascular resistance vessels is influenced by cerebral autoregulation. Capillary and venous blood is contained in a compliance of $\mathrm{C}_{\mathrm{v}}$. Finally, venous blood flows out to the sagittal sinus through the bridging veins $R_{b}$. The CSF pathway encompasses CSF formation $\left(I_{f}\right)$, storage in the distensible fluid structures formed by the ventricles and basal cisterns $\left(\mathrm{C}_{\mathrm{i}}\right)$, and reabsorption through the arachnoid granulations $\left(R_{c s}\right)$ to the sagittal sinus.

All parameters and the mathematical description of the model are presented in greater detail in the appendix.

\section{CLINICAL RECORDINGS}

The results of simulations were visually compared with recordings chosen from the studies performed in 82 patients who had been admitted to Addenbrooke's Hospital after moderate and severe head injury (median Glasgow coma scale after resuscitation 6, range 3 to 13). Each patient was mechanically ventilated to maintain a $\mathrm{PaCO}_{2}$ between 3.5 and $4.5 \mathrm{kPa}$. Intracranial pressure was monitored continuously using a fibreoptic transducer (Camino direct pressure monitor, Camino Laboratories, US), inserted into brain perenchyma in the frontal region. Arterial pressure was measured directly in the radial or dorsalis pedis artery and monitored using a bedside monitor (System 8000, S and W Vickers Ltd, UK). The middle cerebral artery (MCA) was insonated daily for periods up to two hours using a PCDop 842 Doppler ultrasound unit (Scimed, Bristol, UK) to record maximal blood flow velocity waveform (FV). Cortical blood flow was monitored in 26 patients using a laser blood flow monitor (MBF3D, Moor Instruments, Axminster,

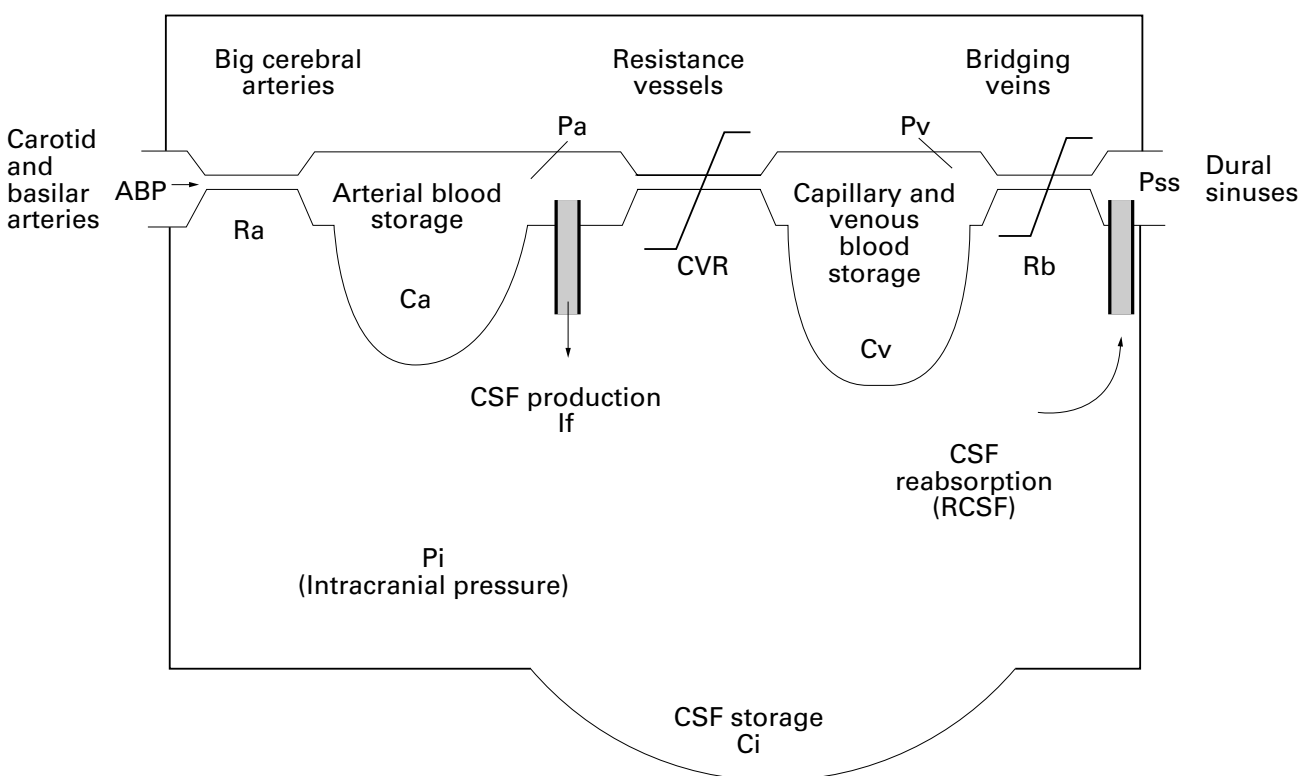

Figure 2 Hydrodynamic equivalent of the model, comprising pathways of CBF and the CSF circulation. A rigid skull is represented by the outer box, with a compensatory reserve $C_{i}$ associated with the compliant dural sac within the lumbar channel. 


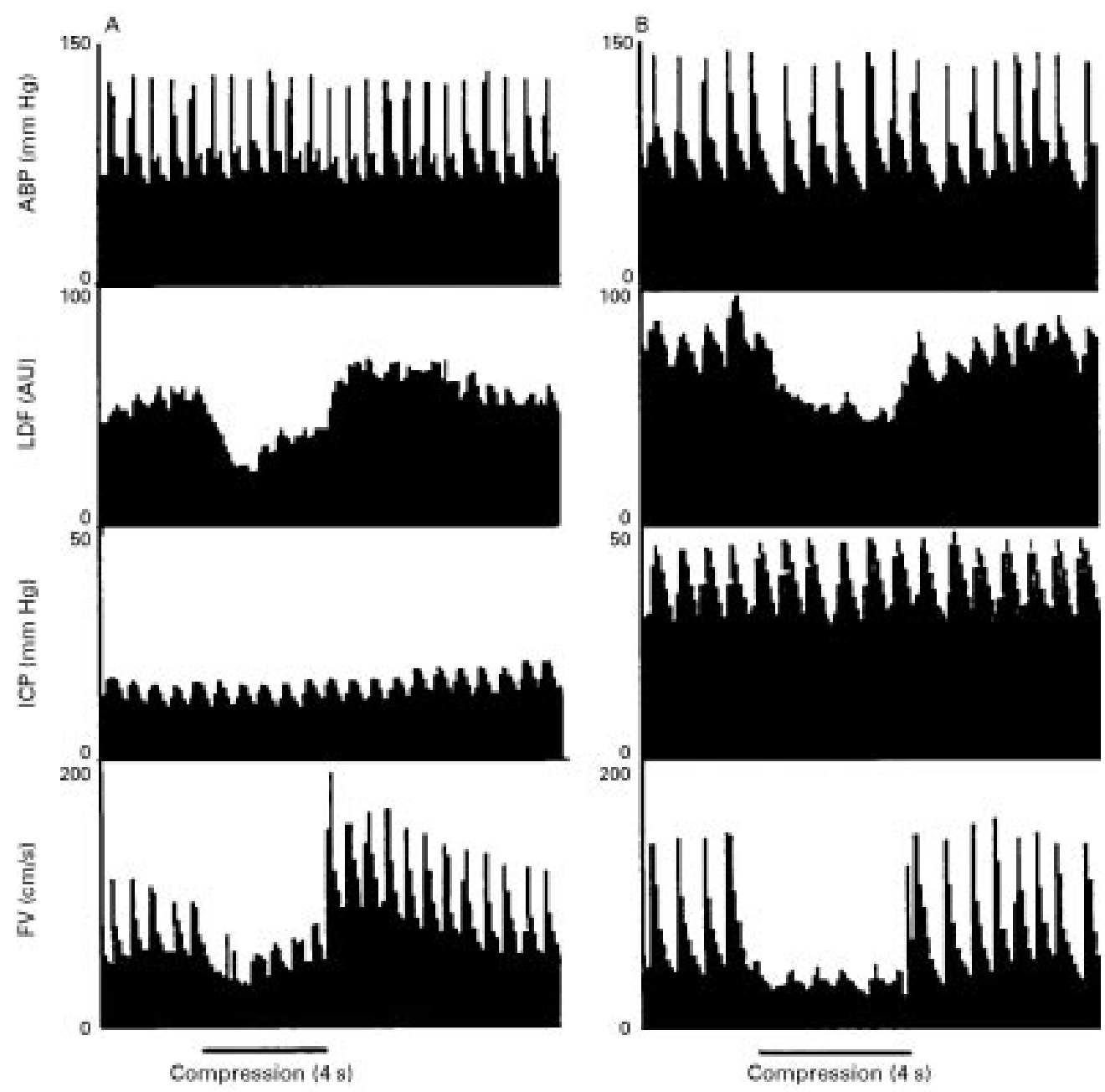

Figure 3 Example of two compression tests performed in patients after head injury. Test $(A)$ shows a positive hyperaemic response in MCA blood flow velocity (FV) and laser Doppler flow meter (LDF) placed on ipsilateral hemisphere observed at CPP>70 mm Hg. Test (B) was performed when ICO increased to $40 \mathrm{~mm} \mathrm{Hg}$, decreasing CPP to around $50 \mathrm{~mm} \mathrm{Hg:}$ there was no hyperaemia. Arterial pressure $(A B P)$ was measured using a central line.

UK); the clinical technique for insertion of the probe has been described. ${ }^{28}$ Short term common carotid artery compression (five to six seconds) was performed to assess cerebral autoregulation. ${ }^{29}$ In seven patients we compared the results of two tests performed at either high $(>80 \mathrm{~mm} \mathrm{Hg})$ or low $(<60 \mathrm{~mm} \mathrm{Hg})$ levels of cerebral perfusion pressure (CPP). The events of Lundberg plateau waves of intracranial pressure (ICP) were recorded in eight patients and episodes of transient arterial hypotension (arterial blood pressure $(\mathrm{ABP})<70 \mathrm{~mm} \mathrm{Hg})$ in 15 patients.

COMPARISON BETWEEN MODELLING SIMULATIONS AND CLINICAL RECORDINGS

Changes in transcranial Doppler MCA blood flow velocity (FV) correspond to simulated changes in flow through the great cerebral arteries $(\mathrm{CBF}+)$. Changes in laser Doppler capillary red cell flux (LDF) may be compared with simulated changes in blood flow through resistance vessels (mCBF). Cerebral perfusion pressure $(\mathrm{CPP}=\mathrm{ABP}-\mathrm{ICP})$ corresponds to the simulated gradient $\mathrm{ABP}-\mathrm{P}_{\mathrm{i}}$. However "real" cerebral perfusion pressure $\left(\mathrm{P}_{\mathrm{a}}-\mathrm{P}_{\mathrm{v}}\right)$ cannot be measured clinically.

\section{Results}

SHORT TERM HYPOPERFUSION: TRANSIENT HYPERAEMIC RESPONSE TEST

The recordings made in patients after head injury were compared with the mathematical simulations. In every clinical instance when $\mathrm{CPP}$ was $>70 \mathrm{~mm} \mathrm{Hg}$ (fig 3 (A)) after four to six seconds CCA compression the hyperaemic response was visible on TCD (laser Doppler recordings were performed in two patients). By contrast, in the same patients when CPP fell below $50 \mathrm{~mm} \mathrm{Hg}$ due to intracranial hypertension (ICP>30 $\mathrm{mm} \mathrm{Hg}$ ) the positive autoregulatory response disappeared (fig 3 (B)).

A response to compression was simulated by a short term increase in the resistance of the big cerebral arteries $\left(R_{a}\right)$ from 0.01 to 0.08 $\mathrm{mmHg} /(\mathrm{ml} / \mathrm{min})$. Simulation was conducted with intact autoregulation and with autoregulation impaired (by equalising the levels of maximal vasodilatation and maximal vasoconstriction to the value of $0.2 \mathrm{~mm} \mathrm{Hg} /(\mathrm{ml} / \mathrm{min}))$. In both cases a significant reduction of simulated cerebral perfusion pressure $\left(C P P=P_{a}-P_{v}\right)$ was recorded during compression. In the autoregulating system the cerebral vessels dilated, which was shown as a gradual decrease in CVR with 

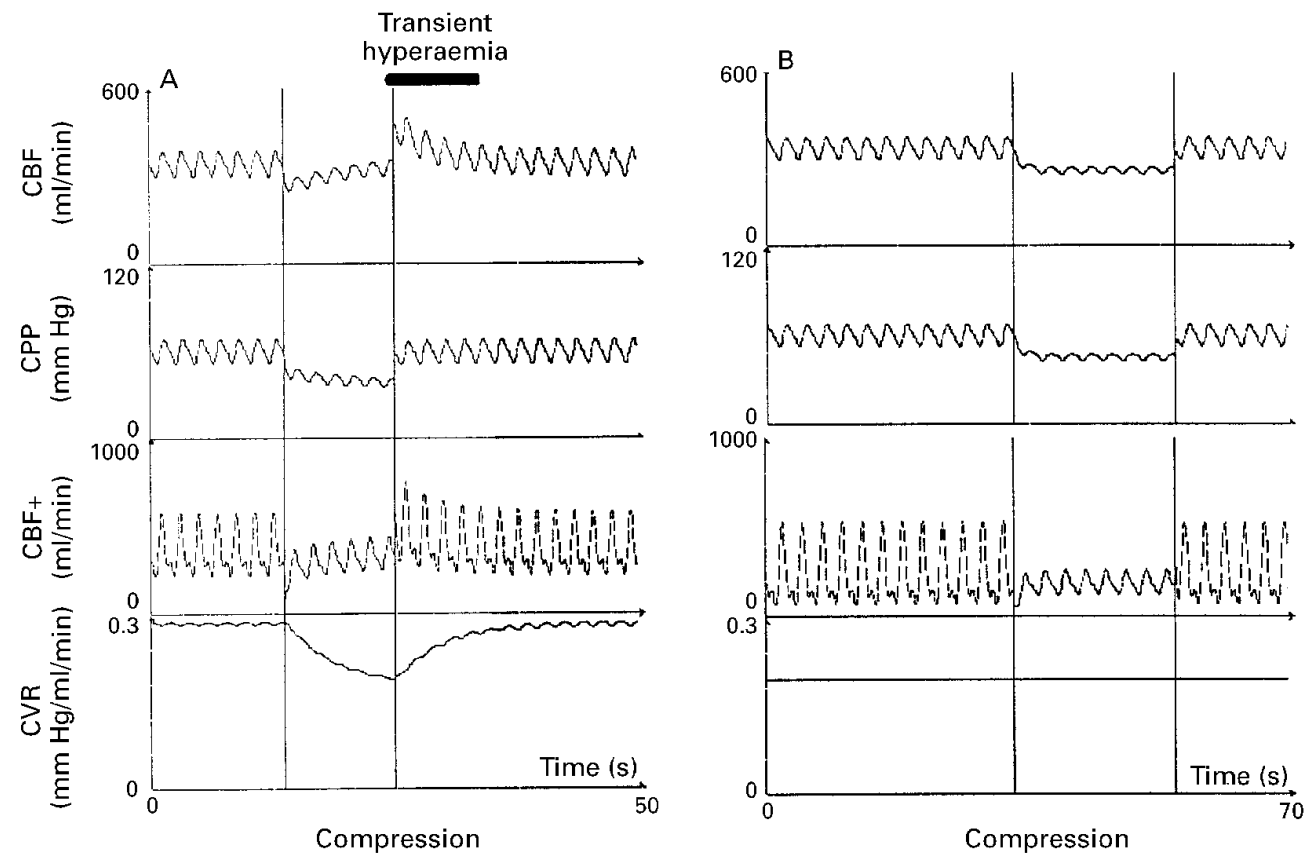

Figure 4 Dynamic response to a simulated seven second compression of the common carotid artery. $C B F=$ flow through small cerebral vessels; $C B F+=$ flow through big cerebral vessels; $C V R=$ the resistance of small cerebral vessels. Compression causes transient hyperaemia in the autoregulating system $(A)$ and a passive recovery of the flow to the baseline in the non-autoregulating system (B)

a time constant equivalent to the delay of autoregulation. The model indicated that blood flow through the big cerebral arteries $(\mathrm{CBF}+)$ and arterioles $(\mathrm{CBF})$ decreased immediately during the compression, and then started to increase, as the cerebral arterioles dilated (fig 4 (A)). After a release of compression, the CPP increased abruptly towards the baseline. As the vessels remained predilated, a transient hyperaemia was recorded until CVR returned to the baseline. When autoregulation was impaired, the reduction in CPP did not induce vasodilatation (CVR remained constant). After the release of compression, CBF and $\mathrm{CBF}+$ returned passively to the baseline (fig 4 (B)) without any overshoot. Hence we can conclude that the postcompression hyperaemia is a reflection of intact autoregulation.

\section{ARTERIAL HYPOTENSION}

During episodes of spontaneous arterial hypotension, when MAP dropped from $>80 \mathrm{~mm} \mathrm{Hg}$ to below $70 \mathrm{~mm} \mathrm{Hg}$ for 10 minutes or more we found a specific haemodynamic response in 15 patients. Figure 5 shows an example in which ABP fell after discontinuation of an infusion of dopamine. Whereas the diastolic flow velocity decreased from the very beginning, the systolic flow velocity increased until point $A$ and then started to decrease. The changes in ICP paralleled changes in systolic flow velocity; thus ICP increased until point $A$ and then decreased. Point $\mathrm{A}$, which indicated the break in systolic flow velocity and the beginning of reduction of ICP, was detected below CPP of $55 \mathrm{~mm} \mathrm{Hg}$ in every instance (range $55 \mathrm{~mm} \mathrm{Hg}$ to $37 \mathrm{~mm} \mathrm{Hg}$ ).

We used our model to simulate the effect of a gradual decrease in arterial blood pressure according to the cerebrovascular system au- toregulating (fig 6 left or non-autoregulating (fig 6 right). In an autoregulating system, the value of arterial $\mathrm{CBF}$ averaged in time (mCBF) was constant until mean CPP decreased below the lower limit of autoregulation (this limit was programmed in the model at 40 $\mathrm{mm} \mathrm{Hg}$ ). A gradual decrease in CPP towards this lower limit translated into a gradual increase in ICP. The systolic flow in large cerebral arteries $(\mathrm{CBF}+)$ increased whereas diastolic $\mathrm{CBF}+$ decreased; thus the net effect was an increase in the pulse amplitude of $\mathrm{CBF}+$. The cerebral arterioles dilated, as indicated by a decrease in CVR, until the vessels at the lower limit of autoregulation became dilated maximally at the time point $\mathrm{A}$-corresponding to time point $\mathrm{A}$ in fig 5 . Below this point, further decrease in $\mathrm{ABP}$ led to a decrease in mean $\mathrm{CBF}$ and ICP. Coincidentally with the mean CPP reaching the lower limit of autoregulation, the diastolic CPP started to fall below the critical closing pressure. This was indicated by a momentary pulsatile increase in CVR, initially for a short part of each pulse, and then during most of the pulse cycle. The diastolic CBF+ fell to zero as the arterioles started to close intermittently. Finally, when the systolic CPP fell below the lower limit of autoregulation, the systolic $\mathrm{CBF}+$ started to decrease.

By contrast, when we modelled nonautoregulating cerebrovascular systems, all variables behaved more uniformly, without visible breakpoints. Thus when ABP decreased, both arteriolar CBF and ICP fell. Systolic and diastolic $\mathrm{CBF}+$ each decreased and did not show the divergent behaviour seen in the autoregulating system. The pulse amplitude of $\mathrm{CBF}+$ did not change until it began to fall 


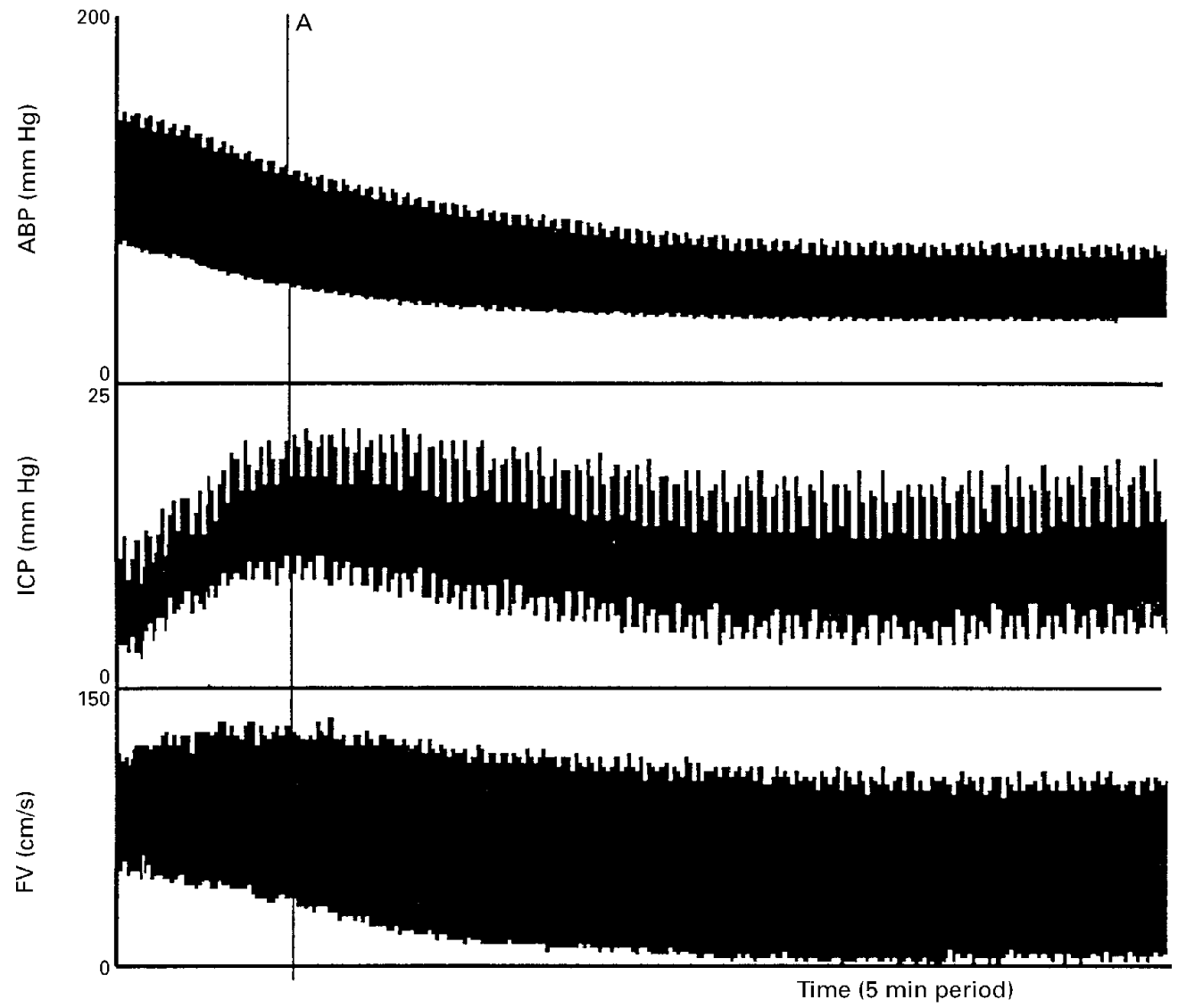

Figure 5 An episode of transient arterial hypotension recorded in a patient with head injury. Arterial pressure (ABP), intracranial pressure (ICP), and the MCA blood flow velocity were recorded. Point $A$ denotes switching point below systolic FV and ICP started to decrease.

when the diastolic CPP reached the critical closing level.

Using both clinical observation and modelling data we can conclude that in patients with gradual reduction of $\mathrm{ABP}$, leading to a decrease in CPP, maintained systolic FV, decreasing diastolic FV, and the increase in ICP indicates preserved autoregulation.

\section{INTRACRANIAL HYPERTENSION}

Episodes of spontaneous intracranial hypertension were recorded in seven patients. Figure 7 shows a provoked haemodynamic response that was similar in each patient. An increase in ICP above $30 \mathrm{~mm} \mathrm{Hg}$ was followed by a gradual decrease in diastolic $\mathrm{FV}$ and an increase in systolic FV. After point A (when ICP was from 30 to $45 \mathrm{~mm} \mathrm{Hg}$, and CPP was $<50 \mathrm{~mm} \mathrm{Hg}$ ) systolic $\mathrm{FV}$ ceased to increase. After point B (CPP below $35 \mathrm{~mm} \mathrm{Hg}$ ) systolic $\mathrm{FV}$ started to decrease. The average separation between ICP level corresponding to points A and $\mathrm{B}$ was $17 \mathrm{~mm} \mathrm{Hg}$ (range 10 to $22 \mathrm{~mm} \mathrm{Hg}$ ).

An increase in intracranial pressure was modelled as a response to an external infusion of CSF with a stepwise increase in rate. When we simulated this change in the model, in addition to the parameters analysed for systemic hypotension, we included the compliance of the large cerebral arteries (Ca) (fig 8).

The CPP fell with increasing ICP. The amplitude of the ICP pulsations and the com- pliance of the big cerebral arteries increased as both the arteriolar resistance and the amplitude of the CPP pulsations decreased. The time average $\mathrm{CBF}+$ was constant reflecting the systolic value increasing and the diastolic slightly decreasing. The arteriolar blood flow $(\mathrm{mCBF})$ was maintained constant until the mean CPP decreased below the lower limit of autoregulation at time point $\mathrm{C}$-corresponding to the breakpoint A on figure 7. Just below the lower limit of autoregulation, the diastolic CPP decreased below the critical closing level. This was reflected in the CVR starting to pulsate, indicating transient arteriolar closure during diastole. The diastolic $\mathrm{CBF}+$ decreased to zero. The compliance $\mathrm{C}_{\mathrm{a}}$ was maintained because the pressure in the large cerebral arteries was greater than in the cerebral arterioles. Beyond point B, the CPP became too low to keep the big arteries open thus the compliance $\mathrm{C}_{\mathrm{a}}$ started to fall, causing a steep decrease in the systolic $\mathrm{CBF}+$ and the pulse amplitude of the ICP waveform started to decrease.

In the non-autoregulating system (fig 8 right) the divergence of the systolic and diastolic $\mathrm{CBF}+$ specific for intact autoregulation was not seen. The arteriolar CBF, and the systolic, and diastolic $\mathrm{CBF}+$ each decreased as CPP decreased and the cerebrovascular resistance and compliance of the big arteries did not 

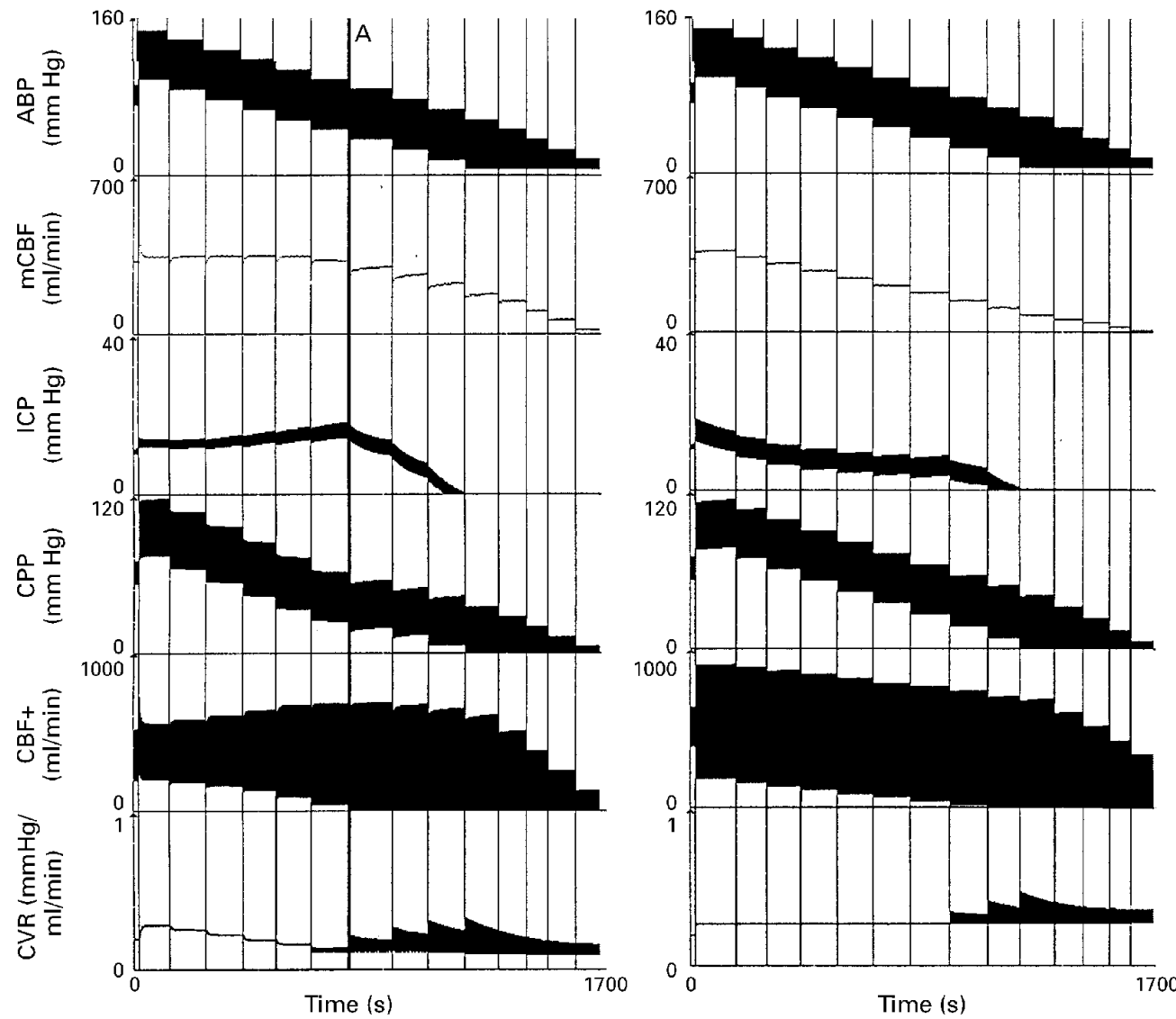

Figure 6 Effect of stepwise decrease in ABP was modelled in autoregulating (left) and non-autoregulating (right) cerebrovascular systems. Vertical line A shows limit of autoregulation; other lines show moments of step changes in arterial pressure $(A B P) ; m C B F=$ time average cortical blood flow; ICP=intracranial pressure; $C P P=$ cerebral perfusion pressure; $C B F+=$ pulsatile blood flow through big cerebral arteries; $C V R=$ cerebrovascular resistance.

change. A breakpoint was detected, (similar to the time point B in the autoregulating system) below which transient closure of arterioles and a gradual decrease in the compliance of big arteries were found.

During gradual intracranial hypertension rising systolic flow velocity with stable or decreasing diastolic flow velocity indicates preserved autoregulation.

\section{Discussion}

These results of mathematical simulations help the interpretation, in terms of preservation of autoregulatory capacity, of the haemodynamic responses to short and long lasting episodes of hypoperfusion that have been seen clinically $^{181930}$ and produced experimentally. ${ }^{11-1431}$ Changes in CBF through the big arteries can be measured indirectly using $\mathrm{TCD},{ }^{8}$ assuming that the cross sectional area of the artery insonated by the probe is constant. Cortical blood flow can be measured using laser Doppler flowmetry in the laboratory ${ }^{1-14}$ and in clinical practice ${ }^{28}$ with very good time resolution, but at only one small location. More reliable ICP transducers are now available. With more refined techniques for clinical measurement, mathematical modelling of the data becomes an important supplement for interpretation of the phenomena recorded.
SHORT TERM HYPOPERFUSION: CAROTID ARTERY COMPRESSION TEST

Carotid artery compression was introduced as a test of cerebral autoregulation many years ago and has been recently revived. ${ }^{72} 3233$ Patients with atheroma of the carotid artery should not be examined using this method, therefore we performed a careful carotid artery Doppler study before the test, to exclude patients not suitable for compression.

A transient hyperaemia after compression and release of the common carotid artery is regarded as evidence of intact autoregulation. $^{72}$ The extent of the hyperaemic overshoot is proportional to the extent decrease in CVR during compression, and can be related to the gradient of the autoregulation curve (fig 9 (C)). However, the hyperaemic response is also related to the decrease in cerebral perfusion pressure during compression, which cannot be measured in clinical practice because pressure distal to the compression is not known. Therefore, quantification of transient hyperaemia may be misleading, even if complex computer support is used. ${ }^{33}$ The main difficulty in interpretation of the test is that it produces "yes or no" results, whereas autoregulation is not an "all or none" phenomenon. 


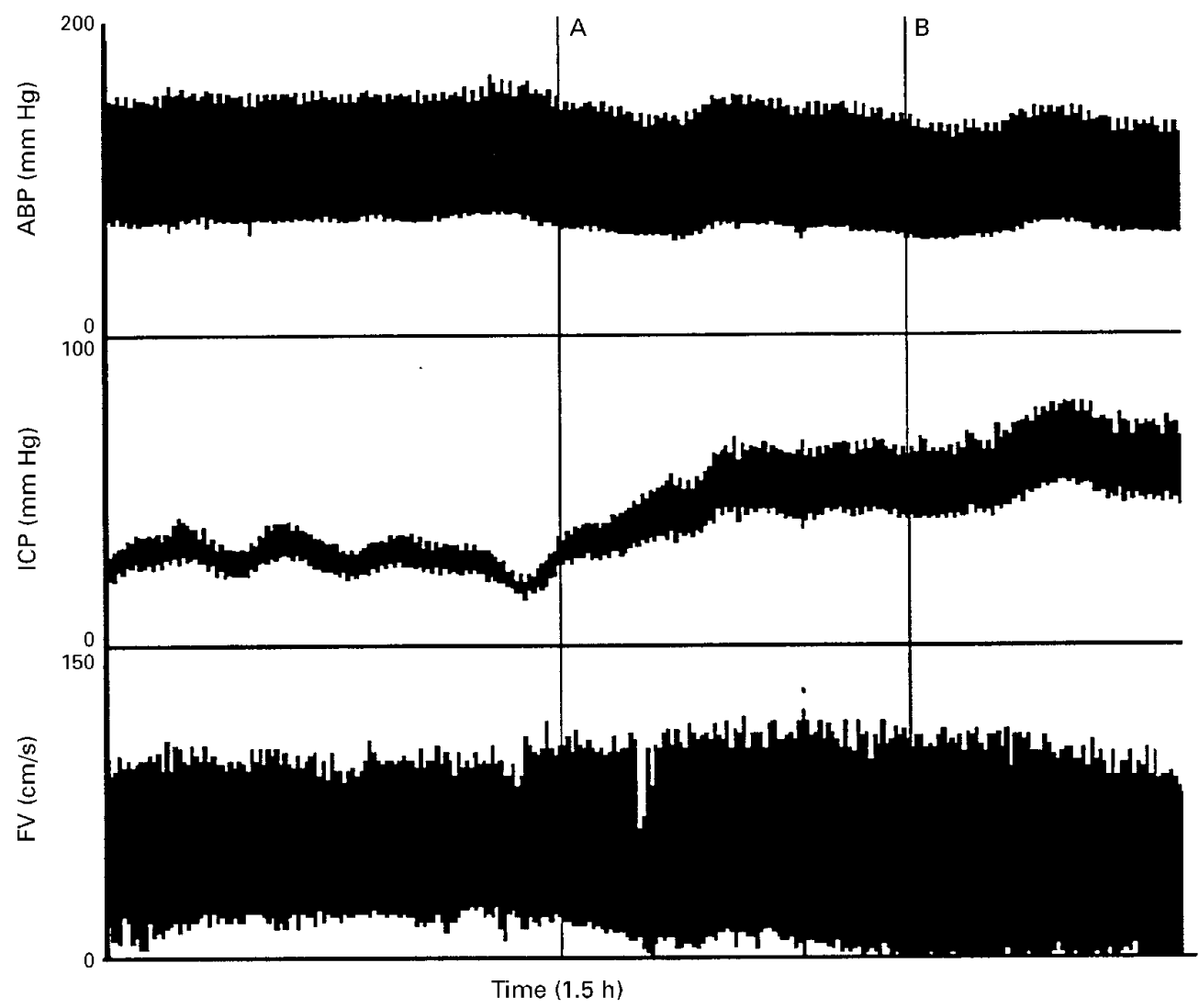

Figure 7 Example of recording of arterial pressure $(A B P)$, blood flow velocity $(F V)$, and intracranial pressure (ICP) during ascending slope of ICP plateau wave. Lines $A$ and $B$ represent the points where systolic $F V$ stopped increasing and started to decrease respectively.

INTERPRETATION OF TCD PULSATILITY INDEX

When there is sustained hypoperfusion due either to intracranial hypertension or to arterial hypotension, our simulations predict that, when autoregulation is intact, the pulsatile component of flow through the big cerebral arteries will increase. Data from clinical observations $^{34}$ and laboratory studies ${ }^{11-13}$ are each consistent with these predicted from our modelling simulations. They have proved that there is need for caution in equating an increasing transcranial Doppler pulsatility index (defined as the ratio of the amplitude of pulsations to the time average value of the blood flow velocity) with exhaustion of autoregulatory reserve. ${ }^{30}$ Our simulations showed that an increase in pulse amplitude of $\mathrm{CBF}+$ occurs in an autoregulating system when CPP is decreasing toward the lower limit of autoregulation. However, the pulsatility index may also increase in non-autoregulating systems, when the $\mathrm{CBF}+$ pulse amplitude remains constant and the time average $\mathrm{CBF}+$ decreases passively. ${ }^{35}$ Recent experimental work $^{31}$ has confirmed that the rise in the pulsatility index when CPP was decreasing could be recorded both with intact autoregulation and in cases when autoregulation was disturbed by previous ischaemic insults.

WAVEFORM ANALYSIS OF TCD

The results of simulation suggest that analysis of changes in the systolic and time average blood flow velocities during spontaneous fluctuations in CPP may enable detection of breakpoints of autoregulation (point A figs 5-8). A negative correlation between changes in CPP and systolic flow velocity, accompanied by a stable time average flow velocity is an indication of "good autoregulation". A pressure passive decrease in time averaged and systolic flow velocities with decreasing CPP signifies impaired autoregulation. An intermediate situation - that is, decreasing CPP accompanied by a falling time averaged flow velocity but a constant systolic flow velocity- marks the state when autoregulation is compromised but still intact. ${ }^{11}$ The results of previous studies in patients with head injury ${ }^{36}$ are compatible with such a conclusion. Such a correlation of fluctuations of CPP with changes in the blood flow velocity waveform can be expressed, using computer waveform analysis, as a time dependent index of the cerebral autoregulatory reserve.

INTRACRANIAL PRESSURE WAVEFORM

Our simulations illustrate that fluctuations in ABP produce inverse changes in ICP when autoregulation is intact. ${ }^{37}$ The correlation becomes positive-that is, ABP and ICP change simultaneously when autoregulation is impaired. Hence, a running correlation coefficient between the time averaged ICP and arterial pressure could be of clinical utility as an index of autoregulatory capacity. ${ }^{38}$ 

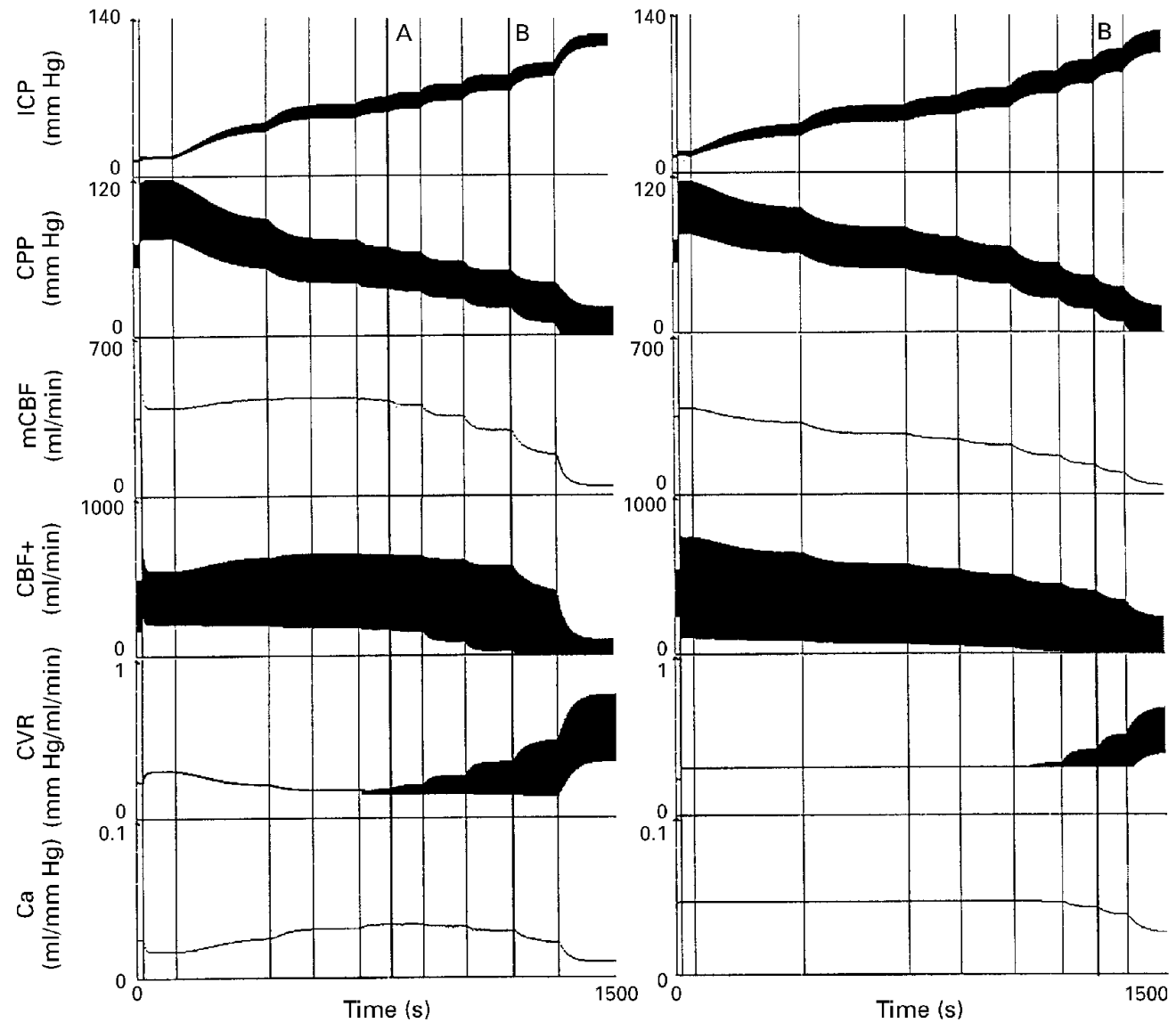

Figure 8 Effect of stepwise increase in intracranial pressure (ICP) was modelled in autoregulating (left) and non-autoregulating (right) cerebrovascular systems. Line A shows limit of autoregulation, line B critical closing of cerebral arteries. Other vertical lines denote changes in the rate of the simulated external infusion. ICP=intracranial pressure; $C P P=$ cerebral perfusion pressure; $m C B F=$ time average cortical blood flow; $C B F+=$ pulsatile blood flow through big cerebral arteries; $C V R=$ cerebrovascular resistance; $C a=$ compliance of big cerebral arteries.

Finally, the relation between the pulse amplitude of the ICP waveform and the time averaged ICP can be used in patients with intracranial hypertension to detect the critical closing phenomenon. When the time average ICP is increasing the decrease in amplitude of the ICP pulsations signifies that the cerebral perfusion pressure has reached the critical closing threshold (point $\mathrm{B}$, fig 8 (B)). This technique has been utilised already in methods for continuous analysis of the intracranial pressure waveform. ${ }^{39}{ }^{40}$

\section{Conclusion}

Our mathematical model helps interpretation of the phenomena that occur when the cerebral haemodynamic reserve is becoming exhausted. A transient hyperaemia after a short term common carotid artery compression, a divergence between the systolic and diastolic blood flow velocity when the cerebral perfusion pressure is decreasing, and/or a negative correlation between time average intracranial and arterial pressures can each be interpreted as indicating intact cerebral autoregulation. Conversely, each of an absent transient hyperaemic response, a pressure-passive behaviour of TCD waveform, and coherent changes in $\mathrm{ABP}$ and ICP slow waves indicate reduced cerebrovascu- lar reactivity to changes in cerebral perfusion pressure.

We thank the Cambridge Overseas Trust for funding the PhD projects for PS and SP and the Raymond Sackler Foundation for supporting PS. MC, SP, and PS are on unpaid leave from the Warsaw University of Technology, Poland. We are very grateful to Professor G Teasdale for his helpful comments.

\section{Appendix: mathematical description of the model}

Figure 2 shows the general structure of the model presented in and discussed in the Methods section. This appendix includes a more detailed description of the model parameters and presents the differential equations used to describe the time dependent relation and internal variables.

\section{ELEMENTS OF THE MODEL}

Resistance of great cerebral arteries $\left(R_{a}\right)$

There are no clear data about any non-linear behaviour of this resistance. It can be permanently increased in the course of arteriosclerotic stenotic disease, during cerebral vasospasm, or increased temporarily-for example, by external compression of the 

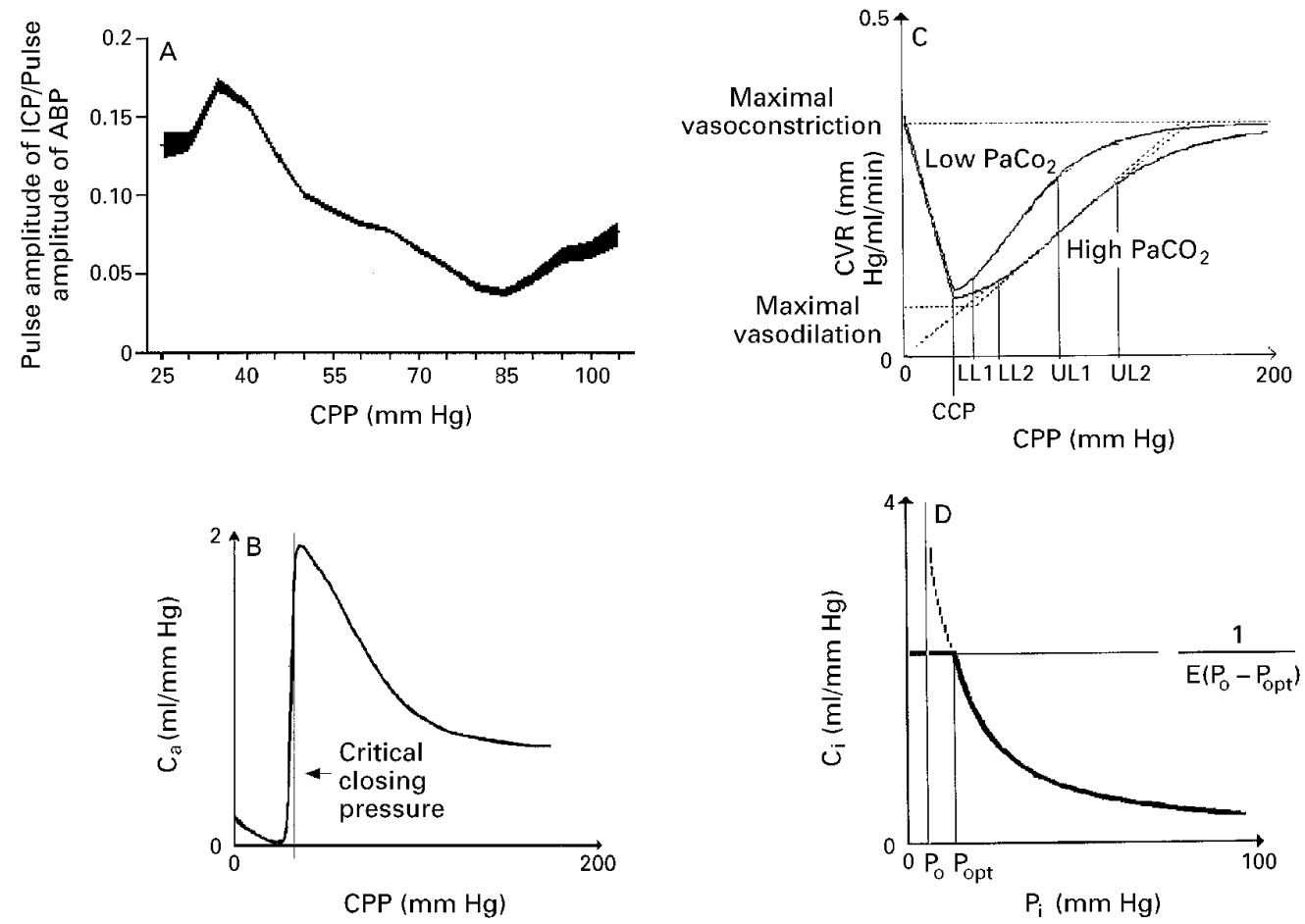

Figure 9 Graphical representation of the most important parameters of the model. (A) Averaged relation between the ratio of the pulse amplitude of ICP to the pulse amplitude of arterial pressure plotted against CPP in 55 patients with head injury. The ratio is theoretically proportional to the compliance of big cerebral arteries $C_{a}$. (B) Modelling relation between $C$ and CPP. (C) Modelling relation between the resistance of small cerebral vessels (CVR) and the CPP, plotted for two different values of arterial $C O$, tension. Region between the lower (LL) and upper (UL) limits of autoregulation represents linear dependence of CVR on CPP. (D) Relation of compliance of CSF space $\left(C_{i}\right)$ with intracranial pressure $\left(P_{i}\right) . P_{\text {opt }}$ represents the so called optimum pressure, above which compliance decreases when ICP increases.

common carotid artery-a manipulation which is discussed in this paper.

Compliance of the great cerebral arteries $\left(C_{a}\right)$ Temporary storage of the inflowing arterial blood in the compliant big arteries is represented by the container $\mathrm{C}_{\mathrm{a}}$. This non-linear compliance plays an important part in the transmission of the arterial blood pulse pressure to the pulsatile pattern of intracranial pressure..$^{10}{ }^{41}$ The ratio of the pulse wave of ICP to the pulse wave of $\mathrm{ABP}$ is theoretically proportional to the compliance $\mathrm{C}_{\mathrm{a}} \cdot{ }^{40}$ The transmission coefficient recorded clinically in patients with head injury and plotted against the cerebral perfusion pressure presents a gradual increase of $\mathrm{C}_{a}$ when CPP decreases, with a maximum at a CPP of around $30 \mathrm{~mm}$ $\mathrm{Hg}$ (fig 9 (A)). This can be explained by a gradual decrease of the basal tone of the big cerebral arteries as CPP decreases (fig 9 (B)). Below the critical closing pressure (CCP) cerebral arteries tend to collapse. ${ }^{42}$ The pulsatile blood volume flowing into the brain decreases, while the pulsatile pressure drive remains almost unchanged; thus $\mathrm{C}_{\mathrm{a}}$ decreases to zero. ${ }^{25}$ The dependence of the $C_{a}$ on arterial gases is poorly documented. In hypercapnia, $\mathrm{C}_{\mathrm{a}}$ probably increases but much less than with arterial hypotension. ${ }^{43} 44$

Cerebral resistance vessels

The next narrowing of the blood flow pathway simulates the CRV constituting the hydrodynamic resistance CVR that is able to accom- modate changes in CPP and respond to the arterial concentration of $\mathrm{CO}_{2}{ }^{2}{ }^{343}$ Figure 9 shows the relation between the CVR and cerebral perfusion pressure. The lower and upper limits of the range where CVR is proportional to CPP correspond to the lower (LL) and upper (UL) limits of cerebral autoregulation. Outside these limits the CVR converges to the horizontal asymptotic values representing the limits of maximal vasodilatation and maximal vasoconstriction. When arterial $\mathrm{CO}_{2}$ increases the CVR decreases, and both LL and UL increase. ${ }^{23} \mathrm{~A}$ programmable delay of the autoregulatory response (range five to nine seconds) is included in the model..$^{38}$ When the CPP decreases below the critical closing pressure (CCP), the CVR starts to increase as arterioles "collapse" with the low transmural pressure. ${ }^{44}$ In the modelling software, the autoregulatory curve may be changed graphically by alteration of the maximal vasodilatation and vasoconstriction levels, the upper and lower limits of autoregulation, the critical closing pressure, and the tension of arterial blood $\mathrm{CO}_{2}$ (fig 9 (C)). The resistance CVR constitutes the functional and anatomical boundary between the high pressure (arterial), and the low pressure (venous) parts of the cerebrovascular bed.

Compliance of capillaries and small veins $\left(C_{v}\right)$ There are no data about its non-linear character. $^{25}$ 


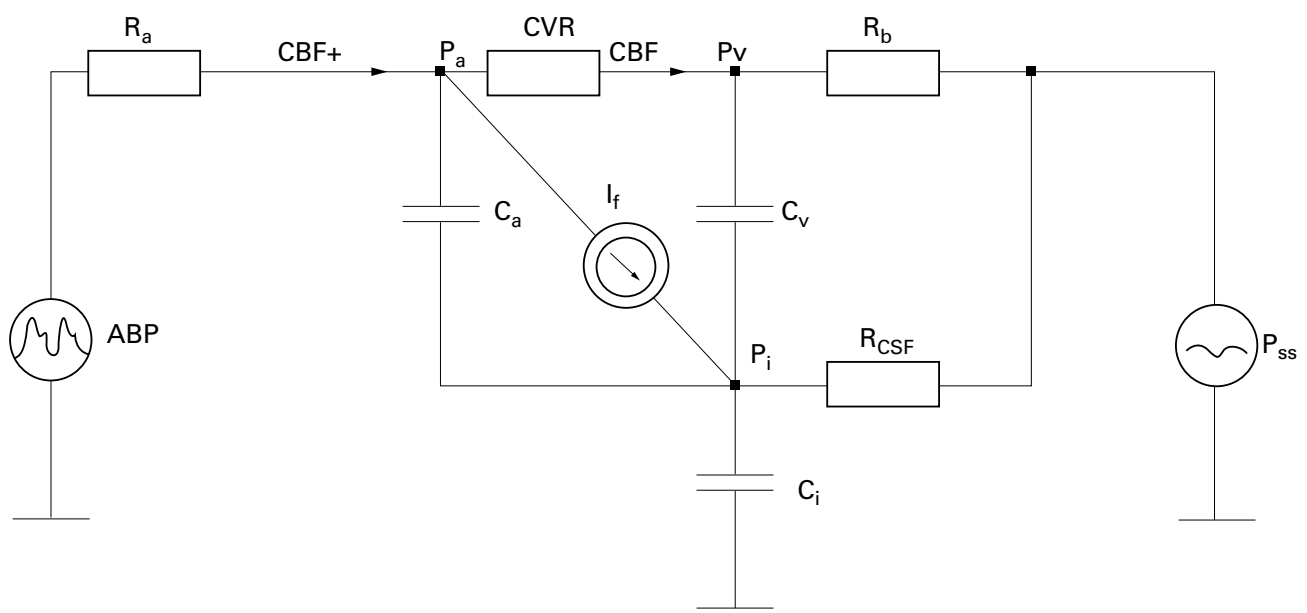

Figure 10 Electrical structure equivalent to the hydrodynamic model (fig 2). All components are described in the text of the appendix.

The resistance of venous outflow $\left(R_{b}\right)$

The venous outflow to the dural sinuses is through the collapsible bridging veins and is represented by a Starling resistor labelled $R_{b}$, controlled by a gradient between intracranial pressure and cerebral venous pressure $\left(P_{v}-P_{i}\right)$. If the gradient becomes negative, $R_{b}$ increases and blocks the venous outflow. If the gradient is positive, $R_{b}$ remains low and relatively constant. This mechanism (which has been questioned in experimental observations ${ }^{45}$ ), together with the CSF circulation, preserves the physiological gradients between the cerebral venous, intracranial, and sagittal sinus pressures. $^{39} 46$

$$
\mathrm{P}_{\mathrm{v}}>\mathrm{P}_{\mathrm{i}}>\mathrm{P}_{\mathrm{ss}}
$$

\section{CSF circulation}

The pathway of the CSF circulation is represented by constant rate secretion of CSF $\left(\mathrm{I}_{\mathrm{f}}\right)$ from the arterial blood, ${ }^{46}{ }^{47}$ storage of CSF inside a cerebrospinal compliance $\mathrm{C}_{\mathrm{i}}$, , and its reabsorption into the dural sinuses through a zero pressure valve of an internal resistance $\mathrm{R}_{\mathrm{csf}}$. Figure 9 (D) shows the relation between the intracranial pressure and CSF compliance. ${ }^{15}{ }^{1620}$ It results from the so-called CSF pressure-volume curve. Below a lower limit, often called the optimal pressure, ${ }^{16}{ }^{17}$ the changes in intracranial pressure are proportional to changes in the intracerebral volume. The compliance, equal to the inverse of the gradient of this curve, is constant. Above the optimal pressure, the increase in the intracerebral volume causes an exponential increase in ICP. ${ }^{20-22}$ Hence, the inverse of its gradient is proportional to the inverse of intracranial pressure measured relative to the reference pressure $P_{\mathrm{o}}$ (fig 9 (D)).

The electrical circuit (fig 10) corresponds directly to the hydrodynamic structure (fig 2). It is used for simulation of the phenomena induced by changes in ABP. The two input voltage sources represent $\mathrm{ABP}$ and venous pressure in the dural sinuses $P_{s s}$. For further analysis of the response to defined excitations, the electrical circuit was described by a set of differential non-linear equations:

$$
\begin{aligned}
& \frac{d P_{i}}{d t}=\frac{1}{C_{i}} \cdot\left(\frac{A B P-P_{a}}{R_{a}}-\frac{P_{i}-P_{s s}}{R_{c s f}}-\frac{P_{v}-P_{s s}}{R_{b}}\right) \\
& \frac{d P_{v}}{d t}=\frac{d P_{1}}{d t}+\frac{1}{C_{v}} \cdot\left(\frac{P_{a}-P_{v}}{C V R}-\frac{P_{v}-P_{s s}}{R_{b}}\right) \\
& \frac{d P_{a}}{d t}=\frac{d P_{1}}{d t}+\frac{1}{C_{a}} \cdot\left(\frac{A B P-P_{a}}{R_{a}}-\frac{P_{a}-P_{v}}{C V R}-I_{f}\right)
\end{aligned}
$$

In the above equations $t$ is a time and $d x / d t$ denotes the first order derivative of the variable $\mathrm{x}$ versus time.

The "state variables" $P_{a}, P_{i}$, and $P_{v}$ together with input ABP, $P_{s s}$ and model parameters permit calculation of the other simulated variables. The program for the IBM PC compatible computer has been designed. ${ }^{35}$ Numerical solving of these equations requires an IBM PC computer (486 or Pentium processor) with a hard disk space of at least $5 \mathrm{MB}$.

\section{Selected abbreviations and acronyms:}

$\mathrm{ABP}=$ arterial blood pressure (measured or simulated)

ICP, $P_{i}=$ intracranial pressure measured (first) and simulated (second)

$\mathrm{CPP}=$ cerebral perfusion pressure

$\mathrm{FV}=$ transcranial Doppler maximal blood flow velocity in the MCA

$\mathrm{LDF}=$ laser Doppler cortical red cell flux

$\mathrm{CBF}=$ simulated blood flow through the cerebral resistance vessels

$\mathrm{CBF}+=$ simulated blood flow through the great cerebral arteries

$\mathrm{P}_{\mathrm{a}}=$ cerebral arterial pressure in the small arteries

$\mathrm{P}_{\mathrm{ss}}=$ sagittal sinus pressure

$\mathrm{P}_{\mathrm{v}}=$ pressure in the cortical veins

$\mathrm{R}_{\mathrm{a}}=$ resistance of great cerebral arteries

$\mathrm{CVR}=$ cerebrovascular resistance controlled by autoregulation

$\mathrm{C}_{\mathrm{a}}=$ compliance of the great cerebral arteries

$\mathrm{C}_{\mathrm{i}}=$ compliance of the CSF containers

$\mathrm{R}_{\mathrm{b}}=$ resistance of the cortical veins and

bridging veins

$\mathrm{R}_{\mathrm{csf}}=$ resistance to CSF outflow

$\mathrm{PaCO}_{2}=$ tension of $\mathrm{CO}_{2}$ in arterial blood

$\mathrm{CCP}=$ critical closing pressure 
1 Miller JD, Becker DP. Secondary insults to the injured brain fR Coll Surg Edinb 1982;27:292-8.

2 Harper $M$, Glass HI. Effect of alterations in the carbon dioxide tension on the blood flow through the cerebral cortex at normal and low blood pressures. $\mathcal{F}$ Neurol Neurosurg Psychiatry 1965;28:449-452.

3 Paulson OB, Strandgaard S, Edvinsson L. Cerebral autoregulation. Cerebrovasc Brain Metab Rev 1991;2:16192.

4 Enevoldsen EM, Jensen FT. Autoregulation and $\mathrm{CO}_{2}$ responses of cerebral blood flow in patients with severe head injury. F Neurosurg 1978;48:689-703.

5 Cold GE, Jensen FT. Cerebral autoregulation in unconscious patients with brain injury. Acta Anaesthesiol Scand 1978;22:270-80.

6 Czosnyka M, Kirkpatrick PJ, Pickard JD. Multimodal monitoring and assessment of cerebral haemodynamic reserve after severe head injury. Cerebrovasc Brain Metab Rev 1996; 8:273-95.

7 Giller CA. A bedside test for cerebral autoregulation using transcranial Doppler ultrasound. Acta Neurochir (Wien) 1991;108:7-14.

8 Aaslid R, Lindegaard KF, Sorteberg W, Nornes H. Cerebral autoregulation dynamics in human. Stroke 1989;20:45-52.

9 Diehl RR, Linden D, Lucke D, Berlit P. Phase relationship between cerebral blood flow velocity and blood pressure.
clinical test of autoregulation. Stroke 1995;26:1801-4.

10 Portnoy HD, Chopp M, Branch C, Shannon M. Cerebrospinal fluid pulse waveform as an indicator of cerebral autoregulation. I Neurosurg 1982;56:666-78.

11 Nelson RJ, Czosnyka M, Pickard JD, Maksymowicz W, Perry S, Martin JL, Lovick AHJ. Experimental aspects of cerebrospinal haemodynamics: the relationship between blood flow velocity waveform and cerebral autoregulation. Neurosurgery 1992;31:705-10.

12 Ungersbock $\mathrm{K}$, Tenckhoff D, Heimann A, Wagner W, Kempski OS. Transcranial Doppler and cortical microcirculation at increased intracranial pressure and during the Cushing response: an experimental study on rabbits. NeuCushing response: an experin
rosurgery 1995;36:147-57.

13 Czosnyka M, Richards H, Kirkpatrick P, Pickard J Assessment of cerebral autoregulation using ultrasound
and laser doppler waveforms - an experimental study in and laser doppler waveforms - an experimental stu
anaesthetized rabbits. Neurosurgery 1994;35:287-93.

14 Richards HK, Czosnyka M, Kirkpatrick P, JD Pickard. Estimation of laser Doppler flux biological zero using basilar artery flow velocity in the rabbit. Am F Physiol 1995;268. H213-7.

15 Marmarou A. A theoretical and experimental evaluation of the cerebrospinal fluid system [thesis]. Drexel University, 1973.

16 Sliwka S. A clinical system for the evaluation of selected dynamic propertiesof the intracranial system [PhD thesis]. Warsaw: Polish Academy of Sciences, 1980. (In Polish.)

17 Avezaat CJ, Eijndhoven JHM. Cerebrospinal fluid pulse pressure and cramiospinal dynamics. [thesis]. Rotterdam: Eressure and craniospinal

18 Langfitt TW, Weinstein JD, Kassell NF. Cerebral vasomotor paralysis produced by intracranial pressure. Neurology 1965;15:622-41.

19 Rosner MJ, Becker DP. Origin and evolution of plateau waves: experimental observations and theoretical model. $f$ Neurosurg 1984;60:312-424.

20 Lofgren J, Zwetnow NN. Cranial and spinal components of the cerebrospinal fluid pressure-volume curve . Acta Neurol Scand 1973;49:575-85.

21 Ekstedt J. CSF hydrodynamic studies in man. $f$ Neurol Neu rosurg Psychiatry 1978;41:345-56.

22 Lofgren J. Mechanical basis of the CSF pressure-volume curve. In: N Lundberg, U Ponten, M Brock, eds. ICP II. Berlin: Springer-Verlag, 1975:79-81.

23 Borgesen SE, Albeck MJ, Gjerris F, Czosnyka M. Computerized infusion test compared to conventional lumbarventricular perfusion for measurement of resistance to CSF outflow. Acta Neurochir (Wien) 1992;199:12-6.

24 Czosnyka $M$, Whitehouse H, Smielewski P, Simac S, Pickard JD. Testing of cerebrospinal compensatory reserve in shunted and non-shunted patients: a guide to interpretation based on observational study. $7 \mathrm{Neurol} \mathrm{Neu}$ interpretation based on observation
rosurg Psychiatry 1996;60:549-58.

25 Hoffmann O. Ein Matetisches Modell zur Simulation und Analyse der intrakraniellen Liquor un Hamodynamik. Habilitationsschrift. Giesen: Justus-Liebig-Universitat, 1985.
26 Sorek S, Bear J. Models of cerebral system mechanics. Haifa: Technion, Israel Institute of Technology, 1986. (Scientific Technion, Israe

27 Ursino M. A mathematical study of human intracranial hydrodynamics. Part I- The cerebrospinal fluid pulse pressure. Ann Biomed Eng 1988;16:379-401.

28 Kirkpatrick PJ, Smielewski P, Czosnyka M, Pickard JD. Continuous monitoring of cortical perfusion using laser Doppler flowmetry in ventilated patients with head injury. fournal Neurol Neurosurg Psychiatry 1994;57:1382-8.

29 Smielewski P, Czosnyka M, Kirkpatrick PJ, Whitehouse H, Pickard JD. Validation of the computerised transient hyperaemic response test as a method for testing autoregulation in severely head injured patients [abstract]. $\mathcal{F}$ Neurotrauma 1995;12:420.

30 Chan KH, Miller DJ, Dearden M, Andrews PJD, Midgley S. The effect of changes in cerebral perfusion pressure upon middle cerebral artery blood flow velocity and jugular bulb venous oxygen saturation after severe brain trauma. $7 \mathrm{Neu}$ rosurg 1992;77:55-61.

31 Whitehouse HE, Richards HK, Czosnyka M, Pickard JD. The discrepancy between the limits of cerebral autoregulation and cerebrovascular resistance during intracrania

32 Czosnyka M, Pickard J, Whitehouse H, Piechnik S. The hypearemic response to a transient reduction in cerebral perfusion pressure-a modelling study. Acta Neurochir (Wien) 1992;115:90-7.

33 Smielewski P, Czosnyka M, Iyer V, Piechnik S, Whitehouse $\mathrm{H}$, Pickard JD. Computerized transient hyperaemic response test - a method for the assessment of cerebral autoregulation. Ultrasound Med Biol 1995;21:599-611.

34 Czosnyka M, Guazzo E, Iyer V, Kirkpatrick P, Smielewski P, Whitehouse $\mathrm{H}$, Pickard JD. Testing of cerebral autoregulation in head injury by waveform analysis of blood flow velocity and cerebral perfusion pressure. Acta Neurochir Suppl (Wien) 1994;60:468-71.

35 Piechnik S, Czosnyka M, Richards H, Kirkpatrick P, Smielewski P, Pickard JD. Effects of decreasing cerebral perfusion pressure on pulsatility of cerebral blood flow velocity - a modelling study. In: Nagai H, Kamiya K, Ishii $\mathrm{S}$, eds. Intracranial pressure IX. Berlin: Springer Verlag, S, eds. Intracra

36 Czosnyka M, Smielewski P, Kirkpatrick P, Menon DK, Pickard JD. Monitoring of cerebral autoregulation in headinjured patients. Stroke 1996;27:829-34.

37 Bouma GJ, Muizelaar JP, Bandoh K, Marmarou A. Bloodpressure and intracranial pressure-volume dynamics in severe head injury - relationship with cerebral blood-flow. $\mathcal{F}$ Neurosurg 1992;77:15-9.

38 Czosnyka M, Smielewski P, Kirkpatrick P, Laing R, Menon D, Pickard JD. Continuous assessment of the cerebral vasomotor reactivity in head injury. Neurosurgery 1997;11: $11-19$.

39 Pickard JD, Czosnyka M. Management of raised intracranial pressure. F Neurol Neurosurg Psychiatry 1993;56:845-58.

40 Czosnyka M, Guazzo E, Whitehouse H, Smielewski P, Czosnyka Z, Kirkpatrick P, et al. Significance of intracranial pressure waveform analysis after head injury. Acta Neurochir (Wien) 1996;138: 531-42.

41 Piper I, Miller JD, Dearden M, Leggate JRS, Robertson I. System analysis of cerebrovascular pressure transmission: an observational study in head injured patients. $\mathcal{F}$ Neurosurg 1990;73:871-80

42 Dewey RC, Pieper HP, Hunt WE. Experimental cerebral hemodynamics. Vasomotor tone, critical closing pressure and vascular bed resistance. $\mathcal{F}$ Neurosurg 1974;41:597-606.

43 Kontos HA, Wei EP, Navari RM, Levasseur JE, Rosenblaum WI, Patterson JL. Responses of cerebrebral arteries and arterioles to acute hypotension and hypertension. Am $\mathcal{7}$ Physiol 1978;234:H371-83.

44 Huber P, Haneda J. Effect of contrast material, hypercapnia, hyperventilation, hypertonic glucose and papaverine on the diameter of the cerebral arteries - angiographic determination in man. Invest Radiol 1967;2:17-32.

45 Auer L, Ishiyama N. Effect of intracranial pressure on bridging veins in rats. F Neurosurg 1987;67:263-8.

46 Davson $H$. Formation and drainage of the CSF in hydrocephalus. In: K Shapiro, A Marmarou, H Portnoy, eds. Hydrocephalus. New York: Raven Press, 1984;112-60.

47 McComb JG. Recent research into the nature of cerebrospi-
nal fluid formation and absorption. $\mathcal{F}$ Neurosurg 1983;59: nal fluid 